\title{
El sendero del cambio estructural en el Bajío: Terciarización económica y digitalización
}

\author{
The path of structural change in the Bajio: Economic tertiarization \\ and digitalization
}

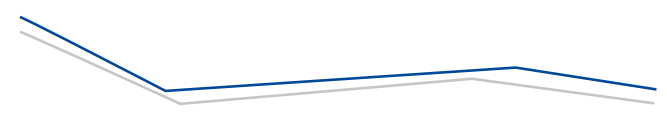

Jordy Micheli-Thirión ${ }^{* \bowtie}$, José Eduardo Valle-Zárate**

Micheli-Thirión, J., \& Valle-Zárate, J. E. (2020). El sendero del cambio estructural en el Bajío: Terciarización económica y digitalización. Investigación y Ciencia de la Universidad Autónoma de Aguascalientes, 28(81), 82-93.

\section{RESUMEN}

El cambio estructural que está teniendo lugar en las economías regionales industrializadas consiste en la creciente importancia que adquiere la terciarización basada en actividades de contenido tecnológico digital. En este artículo se propone una línea de análisis conjunto de la terciarización avanzada y de la brecha digital en los estados de Aguascalientes, Guanajuato y Querétaro, que conforman la región socioeconómica del Bajío. La aportación del texto es caracterizar y comparar el cambio estructural en los tres estados mediante indicadores trabajados con las estadísticas existentes, lo cual permite sugerir un modo de abordaje del fenómeno para otros estados del país.

Palabras clave: brecha digital; TIC; terciarización; cambio estructural; el Bajío.

Keywords: digital gap; $\mathrm{ClT}$; tertiarization; structural change; the Bajio.

\section{Recibido: 6 de agosto de 2019, aceptado: 28 de agosto de 2020}

* Departamento de Economía, Universidad Autónoma MetropolitanaAzcapotzalco. Av. San Pablo 180, col. Reynosa Tamaulipas, C. P. 02200 Azcapotzalco, Ciudad de México, México. Correo electrónico: jordy. micheli@gmail.com ORCID: http://orcid.org/0000-0002-7654-0842

** Programa Economía 4.0, Universidad Autónoma MetropolitanaAzcapotzalco. Av. San Pablo 180, col. Reynosa Tamaulipas, C. P. 02200 Azcapotzalco, Ciudad de México, México.

Correo electrónico: edu_valle3@hotmail.com ORCID: http://orcid. org/0000-0002-4402-3496

Autor para correspondencia

\section{ABSTRAC1}

The structural change taking place in the regional industrialized economies is due to the growing importance of tertiarization based on technological digital activities. In this article we propose a line of joint analysis of both processes for the cases of three Mexican states: Aguascalientes, Guanajuato and Queretaro, that belong to the Bajio socioeconomic region. The contribution of this article is to characterize and compare structural changes in the three states through indicators worked with existing statistics, which suggest a way of approaching the phenomenon for other states of the country.

INTRODUCCIÓN

Los cambios estructurales que suelen identificar a la modernización de la economía están vinculados a la expansión de las tecnologías de información y comunicación (TIC) en las actividades económicas y sociales en general y junto con ello a la expansión de las labores de servicios que perfilan nuevos sectores productivos en la gama de actividades económicas. La sociedad de la información es un proceso convergente con la reestructuración económica basada en terciarización.

Como vislumbró en su momento Bell (1973) y analizó posteriormente Esping-Andersen (1999), dando entrada al concepto de posindustrialización como característica del capitalismo contemporáneo. La historia del concepto de sociedad de la 
información como instrumento geopolítico se encuentra en Mattelart (2009). En ese sentido, la digitalización y terciarización de la economía a escala nacional y regional conforman un proceso integrado cuyo análisis puede orientar el estudio moderno de las economías regionales.

La forma más común de caracterizar la digitalización es mediante índices que recogen diversas dimensiones de la apropiación y potencialidades de apropiación de las TIC por parte de poblaciones en determinados territorios. La comparación interregional del valor de estos índices da paso a la idea de brecha digital, la cual expresa la distancia entre las propias regiones analizadas en materia de digitalización. Por su parte, la terciarización de las economías regionales puede ser dimensionada mediante los datos de la estructura productiva y sus tendencias, tal como hace por ejemplo Garza (2006) para el caso mexicano. Bajo esta premisa es posible generar la información y el análisis que den cuenta de los procesos modernos de cambio estructural.

En este trabajo se propone una línea de abordaje de la reestructuración económica regional bajo la unión de la digitalización y la terciarización, observadas empíricamente con datos que provienen de distintas fuentes del Instituto Nacional de Estadística y Geografía (INEGI). El Bajío es una región social y económicamente conformada en México históricamente (Bataillon, 1969) y aunque la configuración política de esta región abarca a 209 municipios de ocho entidades federativas, hay tres estados que modo integral son parte constitutiva de la región y son el eje económico de la misma: Aguascalientes, Guanajuato y Querétaro.

La escala regional está compuesta por los tres estados del Bajío: Aguascalientes, Guanajuato y Querétaro, mismos que conforman un eje económico de gran dinamismo en México por su integración al modelo industrial-exportador condicionado por el Tratado de Libre Comercio de América del Norte (TLCAN) desde la década de los 90 del siglo pasado.

En una primera parte de este trabajo se muestra el contexto bajo el cual se desarrollan en México la digitalización y la terciarización avanzada. A continuación se brinda una visión estructural de la terciarización de los estados del Bajío, señalando sus semejanzas y diferencias. Finalmente, se explica la metodología y resultados para identificar la brecha digital en México y su caracterización en los estados que nos interesan. El cuerpo de este texto se acompaña de un anexo metodológico en el cual se detalla la forma de calcular el Índice de Desarrollo Tecnológico para México (IDT Mex), con el cual se calcula la brecha digital estatal y municipal.

\section{Terciarizacion y digitalización de las economías regionales}

En la estructura económica actual, el sector terciario ocupa ya un papel dominante (OECD, 2000) y, en consecuencia, para analizar las condiciones del desarrollo local contemporáneo es relevante comprender la emergencia y papel de estos servicios asociados a mayor productividad y uso intensivo y especializado de TIC dentro de la estructura económica regional. Se trata de los servicios avanzados. En el plano cualitativo, resulta conveniente trazar una comparación entre tales servicios y la manufactura, tal como se hace con el caso de México y cuyos resultados más llamativos son los siguientes:

El crecimiento del sector terciario avanzado en México ha sido significativo, pasando de $12 \%$ del PIB terciario en 2003 a $19 \%$ en 2013. Los servicios agrupados y definidos como avanzados se clasificaron como se detalla a continuación. 51: información en medios masivos; 52: servicios financieros y de seguros; 54: servicios profesionales, científicos y técnicos; 55: dirección de corporativos y empresas; 56 : servicios de apoyo a los negocios y manejo de desechos y servicios de remediación (INEGI, 2007).

Debe tomarse en cuenta que si bien la manufactura es de amplia difusión en la geografía nacional ( 14 entidades representan $85 \%$ de la producción manufacturera), en los servicios avanzados tan solo Ciudad de México representa $67.3 \%$ y si agrega Nuevo León, Coahuila y Jalisco se tiene $85 \%$ de representatividad nacional. Sin embargo, en perspectiva temporal ha habido un importante crecimiento del sector de servicios avanzados en los distintos estados; así, a pesar del contexto de desarrollo concentrado de la terciarización en la Ciudad de México se ha producido una expansión de estas actividades a nivel regional y si se compara con la manufactura a escala regional puede decirse que los servicios avanzados han sido más dinámicos. 
En cuanto a la digitalización de las economías, conviene recordar que Brynjolfsson y McAffe (2014) definen como exponencial y combinatoria el avance de la revolución digital en la era actual, y con ello denotan la gran velocidad de los cambios y el carácter convergente de las diversas tecnologías bajo el paradigma del internet de las cosas y de las nuevas capacidades de info-acumulación. Para la CEPAL (2016), la habilidad y posibilidades de adaptación de las naciones a la revolución digital es un nuevo factor de desarrollo que debe ser tomado en cuenta.

La forma más común de observar la digitalización de la economía es la de dimensionar la apropiación de las TIC y comparar los resultados en unidades espaciales significativas: países y regiones. Este ejercicio de conmensurabilidad arroja el concepto de brecha digital, el cual expresa una inequidad social y económica entre la población; en este caso entre la conectada y la no conectada a Internet y, por ende, la usuaria (o potencialmente usuaria) y la no usuaria de las TIC. Toudert (2013) advierte que la brecha digital constituye un nuevo marco intrínseco de relaciones sociales a escala territorial.

La Asociación Mexicana de Internet (AMIPCl, 2016) afirma que en México existen 65 millones de usuarios, con $59 \%$ de penetración y que con esa cifra de usuarios México es el décimo país a nivel mundial. Sin embargo, es un dato que puede tener valor para fines comerciales, pues finalmente el tiempo de consumo de redes es el factor de ganancias para las empresas de telecomunicaciones, pero en realidad tiene una capacidad explicativa menor para asociar digitalización con desarrollo regional. En la figura 1 se ejemplifica la convergencia entre manufactura y digitalización con un auto.

\section{Marco geoeconómico: los tres estados analizados}

Los tres estados contiguos son bastante disímbolos en su extensión territorial. El mayor es Guanajuato con $30,600 \mathrm{~km}^{2}$ y sigue el estado de Querétaro con $11,600 \mathrm{~km}^{2}$, mientras que Aguascalientes abarca tan solo $5,615 \mathrm{~km}^{2}$ (INEGl, 2015). En cuanto al tamaño de su economía, medida a través del PIB per cápita, se encuentra en primer lugar Querétaro, quien es sexto lugar nacional, con $\$ 234,980$; en segundo lugar Aguascalientes con $\$ 214,212$, octavo lugar nacional y en la última posición Guanajuato, con \$154,745, 17 a nivel nacional, debajo de la media nacional.
En el indicador de menor pobreza relativa está Aguascalientes con $28.2 \%$ de la población en condición de pobreza, seguido de Querétaro con $31.1 \%$ y Guanajuato con $42.4 \%$, prácticamente idéntico a la media nacional (El Observatorio Económico México Cómo Vamos, A. C., 2019). En cada estado existe una importante aglomeración urbana con características de zona metropolitana. Con datos de 2015, la mayor es la de León de los Aldamas, en Guanajuato, con 1,714,000 habitantes, en la sexta posición nacional sigue Querétaro con $1,214,000$ personas y en tercer lugar Aguascalientes con 1,017,000 habitantes (Secretaría de Gobernación \& Secretaría de Desarrollo Territorial y Urbano, 2018).

Cada uno de los estados entra a la fase de globalización a partir de condiciones diferentes en su proceso de industrialización, pero con importantes rasgos comunes, ya que desde la década de los sesenta del siglo pasado eran entidades que habían iniciado una incipiente industrialización basada tanto en manufacturas tradicionales como en grandes empresas que deslocalizaban su producción desde la región metropolitana de la capital del país.

La base industrial moderna de estos tres estados se conformó a fines de la década de los sesenta bajo el impulso de políticas locales y en el marco de la última fase del modelo de desarrollo estabilizador, transitando de modo continuo hacia la exportación en el marco de la globalización mediante nuevas inversiones de empresas multinacionales. Esta modernización industrial del Bajío está documentada e interpretada desde una literatura de economía regional que incluye, entre otros autores, a DavilleLandero (2012), Gutiérrez Castorena y Gutiérrez Castorena (2006), Micheli Thirión (2019), Salmerón Castro (1998) y Unger-Rubín (2011).

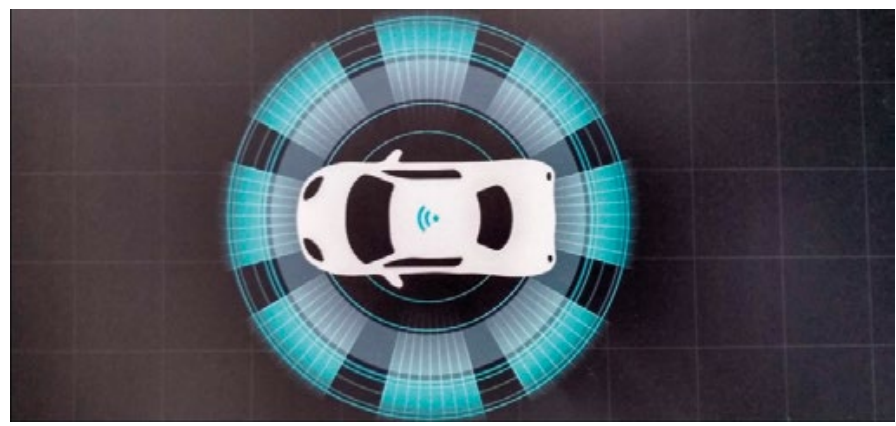

Figura 1. Pictograma. El auto conectado a Internet representa la convergencia entre manufactura y digitalización. Su producción y uso en la región del Bajío será realidad en un futuro cercano. Fotografía de los autores sobre bocetos. 
Terciarización avanzada en las economías del Bajío

La inversión extranjera directa (IED) ha sido sin duda el motor de la industrialización orientada a la exportación en los tres estados del Bajío desde fines del siglo pasado. En conjunto, dichos estados han sido receptores de $8.4 \%$ de la IED recibida por México en el sector de manufacturas durante el periodo $1999-2017$ y $22.8 \%$ de la IED en la rama automotriz. Sin embargo, la región no ha mostrado cambios relevantes en su participación nacional como productora de manufacturas, aunque se pueden advertir modificaciones importantes en la producción de servicios avanzados en los casos de Guanajuato y Querétaro, los cuales multiplican su participación nacional, como se muestra en la tabla 1. Esto indica una evolución económica regional en dos tiempos: lenta en lo industrial y de mayor velocidad en los servicios avanzados, como se ve en la tabla 2.

Tabla 1

Participación de cada estado del Bajío en la producción de manufactura y servicios avanzados nacionales (1999-2013)

\section{Manufactura Servicios Avanzados}

\begin{tabular}{lcccccccc}
\hline Entidad & 1998 & 2003 & 2008 & 2013 & 1998 & 2003 & 2008 & 2013 \\
\hline Aguascalientes & 1.6 & 1.8 & 1.8 & 1.5 & 0.3 & 0.2 & 0.4 & 0.3 \\
Guanajuato & 5.6 & 5.8 & 5.1 & 5.8 & 0.8 & 1.0 & 2.1 & 2.2 \\
Querétaro & 3.5 & 2.6 & 3.3 & 3.0 & 0.4 & 0.7 & 1.1 & 1.0 \\
\hline
\end{tabular}

Nota: Elaboración propia con datos del INEGI $(1999,2004,2009$, 2014).

Tabla 2

Dinamismo de la producción sectorial en cada estado del Bajío (tasa media de crecimiento 1998-2013)

\begin{tabular}{l|c|c|c|c}
\hline Entidad & Manufactura & Servicios & $\begin{array}{c}\text { Servicios no } \\
\text { avanzados }\end{array}$ & $\begin{array}{c}\text { Servicios } \\
\text { Avanzados }\end{array}$ \\
\hline Nacional & 1.74 & 3.56 & 1.28 & 6.82 \\
\hline Aguascalientes & 1.33 & 3.02 & 2.67 & 4.77 \\
Guanajuato & 2.01 & 4.76 & 2.52 & 13.85 \\
Querétaro & 0.70 & 6.26 & 4.51 & 13.06 \\
\hline
\end{tabular}

Nota: Elaboración propia con datos del INEGI $(1999,2014)$.

La mirada a la evolución del peso en la economía nacional que tienen los tres estados se complementa con la de su estructura interna de producción. Como se ilustra en la figura 2, los tres estados mantienen trayectorias convergentes y con tenden- cia a la baja en su componente de manufactura y aumento de los servicios, tanto avanzados como tradicionales; en el caso de los primeros Aguascalientes es el menos dinámico de los tres estados. 


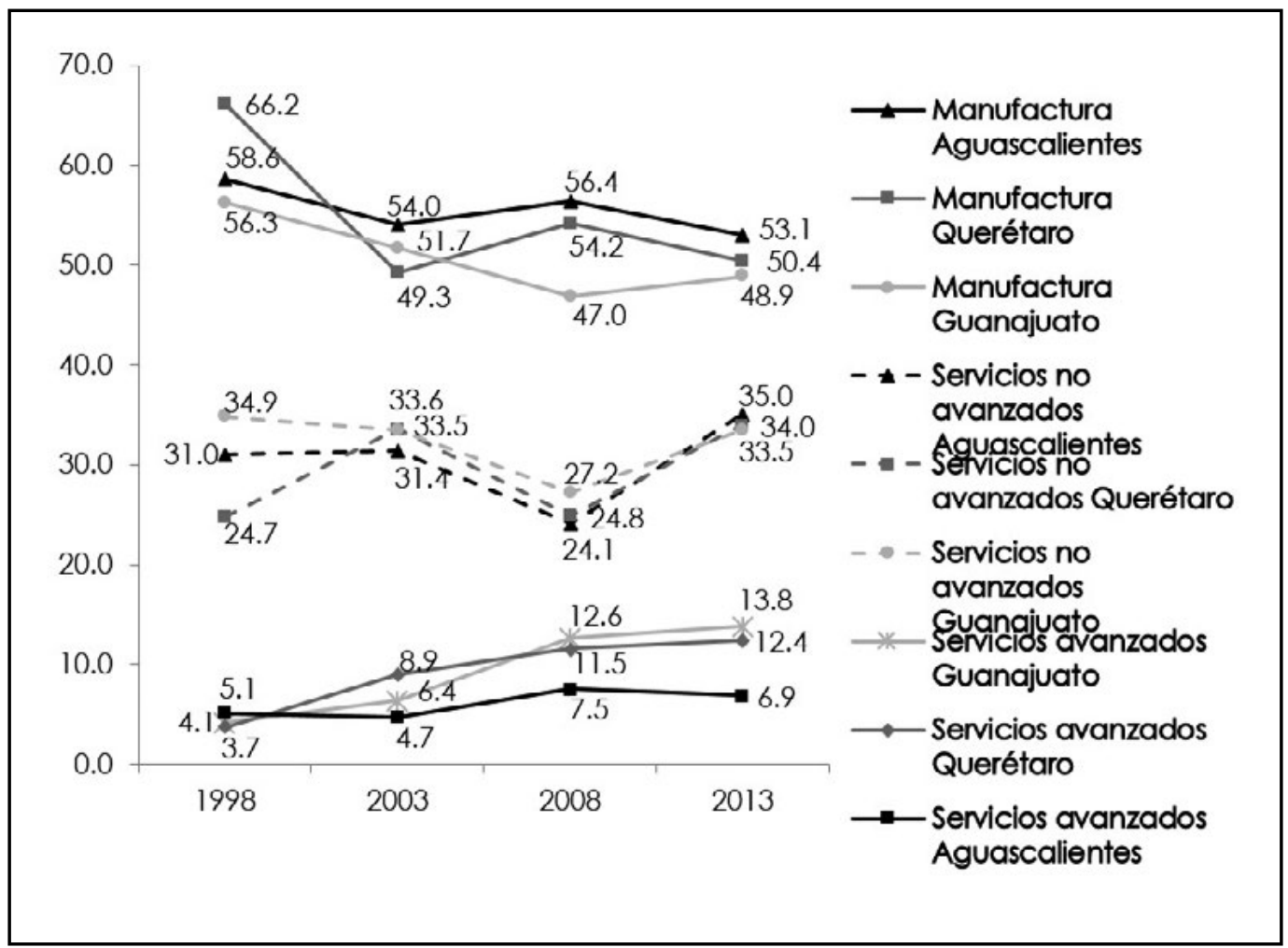

Figura 2. La participación sectorial dentro de cada estado del Bajío (1998-2013).

Elaboración propia con datos del INEGI $(1999,2004,2009,2014)$.

En suma, el sendero de la terciarización avanzada es compartido por los tres estados, aunque con una velocidad notoriamente menor en Aguascalientes y con mayor dinamismo en Querétaro. Llama la atención que a pesar del crecimiento de IED en el sector de manufacturas, únicamente en Guanajuato se registre un dinamismo del producto manufacturero superior al nacional. Si observamos la participación de cada sector dentro de sus respectivos estados, el patrón evolutivo es semejante: baja la manufactura, si bien se mantiene como el sector dominante; suben los servicios avanzados aunque son los de menor peso, y los servicios tradicionales prácticamente mantienen su peso. Esas son las características generales del cambio estructural en los tres estados.

\section{Digitalización en las economías del Bajío}

El nivel de apropiación social de las TIC puede ser evaluado mediante tres dimensiones: acceso a, utilización de y capacidades de uso, según la Unión Internacional de Telecomunicaciones (UIT, 2014). Cada dimensión se forma, a su vez, por distintas características mensurables (ver Anexo). Mayores valores de cada dimensión particular arrojan un índice agregado de apropiación de TIC superior y ello denota un mayor grado de informatización social.

Se aplicó a escala de los estados de México esta metodología, utilizando datos de la Encuesta Nacional sobre Disponibilidad y Uso de Tecnologías de la Información en los Hogares (ENDUTIH) del INEGI (2016). Se procesó la base de microdatos de 80,125 hogares y 292,055 personas que los habitan, por municipio y estado; a fin de contar con los índices equivalentes a los que utiliza la UIT en sus comparaciones internacionales. Se obtienen así resultados para 843 municipios y 32 estados en los tres componentes: acceso, utilización y capacidades. El primero de ellos hace referencia a la infraestructura para el acceso digital tomando a los hogares como unidad de muestreo, en tanto que los dos últimos se refieren al acceso y capacidades de las personas muestreadas. Se ha llamado al índice así construido IDT Mex y la tabla 3 da cuenta de la distribución por estados de dicho índice.

El foco de atención del presente trabajo se encuentra en la emergencia de los servicios avanzados como argumentos de una nueva fase del desarrollo local. Las capacidades en materia de TIC (prácticas 
digitales de las personas y la infraestructura digital disponible para las mismas) tal como las mide este indicador deben ser corroboradas con el aporte de los servicios avanzados al perfil productivo local. Es en este grupo de actividades laborales en el cual las calificaciones digitales generales de las personas se
En línea con el planteamiento central de este trabajo, consistente en visibilizar las tendencias de cambio estructural articuladas en digitalización y terciarización a nivel regional, cabe apuntar que el índice previo muestra una relación positiva

Tabla 3

Brecha digital en México (2015)

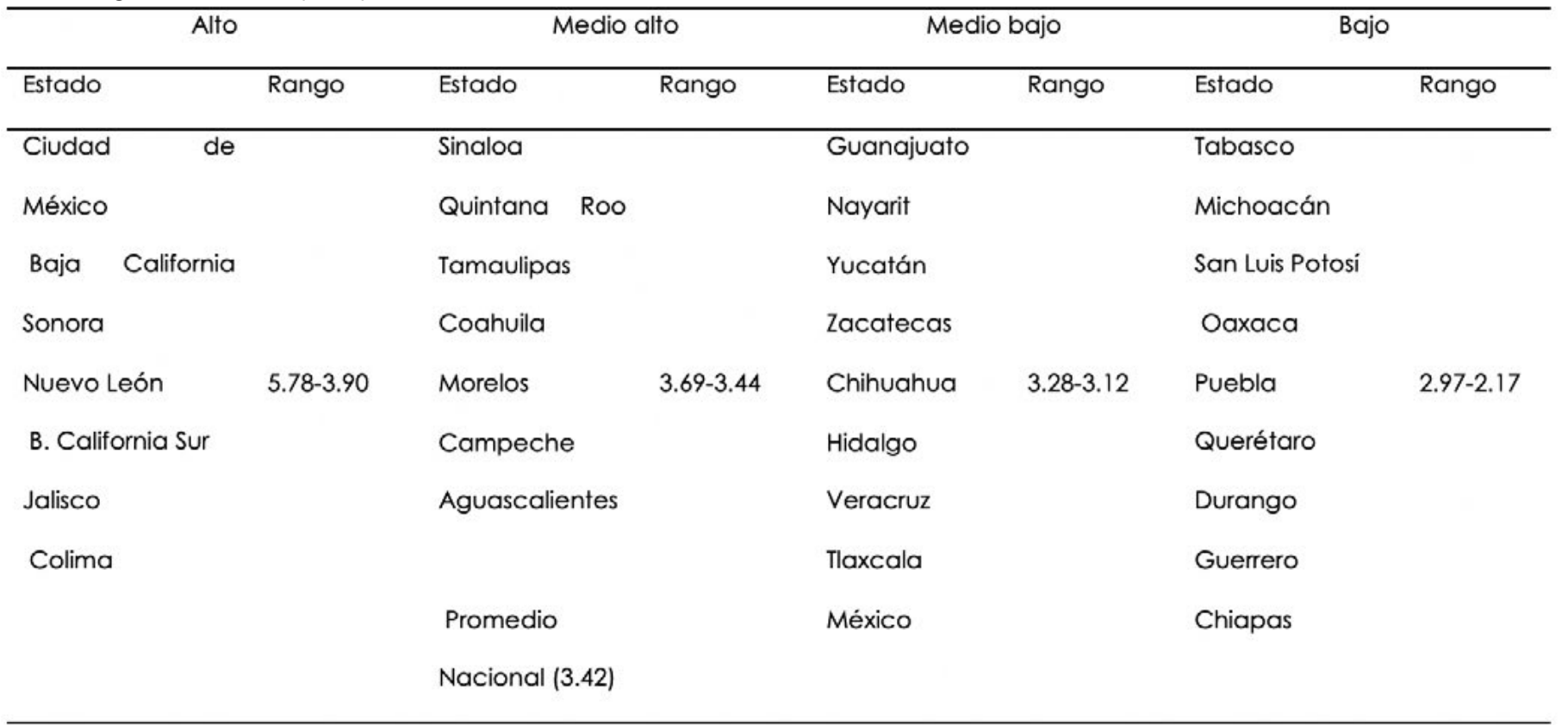

Nota: Los cuatro rangos se definen con base en el promedio nacional y ayudan a una caracterización general de la brecha digital nacional.

Elaboración propia con datos del INEGI (2016).

ponen en acción como parte de su quehacer específico. A falta de estudios de trayectorias formativas y laborales que confirmen y dimensionen esta relación entre competencias digitales y trabajo de servicios avanzados, nuestra propuesta consiste en comparar las curvas de regresión (ajustes por mínimos cuadrados) entre la colección de datos del IDT Mex por estado y la participación de manufactura, servicios y servicios avanzados en la población ocupada total en cada estado, en búsqueda de consistencias entre las variables en lugar de causalidades. Los resultados gráficos se muestran a continuación en la figura 3 y señalan lo siguiente acerca del nivel de apropiación social de las TIC:

-No guarda relación con la manufactura -Está positivamente relacionada con los servicios avanzados

-Está negativamente relacionada con los servicios no avanzados con el sector de servicios avanzados, el de mayor importancia en términos de productividad y de habilidades de trabajo basado en tecnologías digitales. En otras palabras, en México la configuración regional de la brecha digital muestra una relación con el peso de los servicios avanzados en los empleos locales. Por ello, la distribución de los estados que se muestra en la tabla 3 es coincidente en sus posiciones extremas con el peso y el desarrollo socioeconómico de las entidades involucradas (Ciudad de México en un caso, Chiapas y en el otro), pero en el espacio medio es importante tener en cuenta que si bien hay estados de relevancia por su crecimiento reciente, también son relativamente débiles en materia de sociedad de la información.

Como se ha mencionado, el susodicho índice está compuesto a su vez por tres dimensiones: acceso, utilización y capacidades, por lo cual es necesario mostrar esta composición para lanzar una mirada de mayor capacidad explicativa sobre 


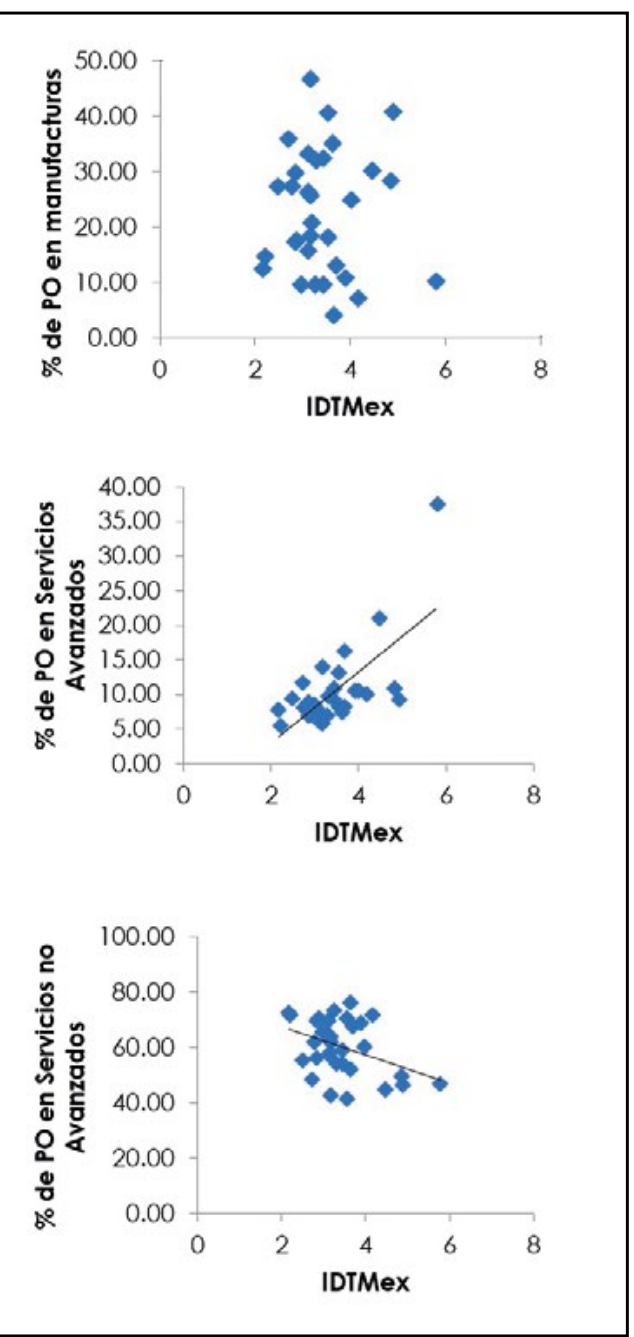

Figura 3. Relación del IDT Mex con la estructura laboral en manufactura, servicios avanzados y servicios no avanzados. PO es población ocupada.

Elaboración propia con datos del INEGI (2016). las características de la digitalización regional en el Bajío. Ello tiene lugar en la tabla 4, el cual señala el dato de cada componente para cada estado, agregando un comentario sobre el sitio que ocupa cada estado en una lista nacional. Como puede verse, esta desagregación del IDT Mex para cada estado confirma que Aguascalientes sobresale de los otros dos estados y Querétaro tiene bajo desempeño en las tres dimensiones.

A continuación se muestra la distribución del índice al nivel de municipios para cada estado. Como se advierte, la distribución geográfica en Querétaro puede brindar una explicación de la aparente contradicción entre ser el estado de mayor dinamismo en servicios avanzados pero el de menores desempeños en digitalización: en efecto, en un solo municipio del estado se localiza una digitalización alta, de modo que la figura 4 muestra un estado de gran polarización territorial, situación que no se presenta en los otros dos estados. En la figura 5 se muestra la estación del ferrocarril de Aguascalientes, clave en la economía del estado hasta inicios de la última década del siglo XX.

Tabla 4

Dimensiones del IDT Mex por cada estado del Bajío (2015)

\begin{tabular}{|c|c|c|c|c|c|c|}
\hline DIMENSIÓN & Aguascalientes & & Guanajuato & & Querétaro & \\
\hline Acceso a las TIC, & cerca & del & cerca & del & 2.77, debajo & del \\
\hline Nacional 3.57 & nacional & & nacional & & nacional & \\
\hline Utilización de TIC, & cerca & del & 2.61, debajo & del & 2.01, debajo & del \\
\hline Nacional 2.76 & nacional & & nacional & & nacional & \\
\hline Capacidades de uso & superior & al & debajo & del & debajo & del \\
\hline de TIC, & nacional & & nacional & & nacional & \\
\hline Nacional 4.45 & & & & & & \\
\hline
\end{tabular}




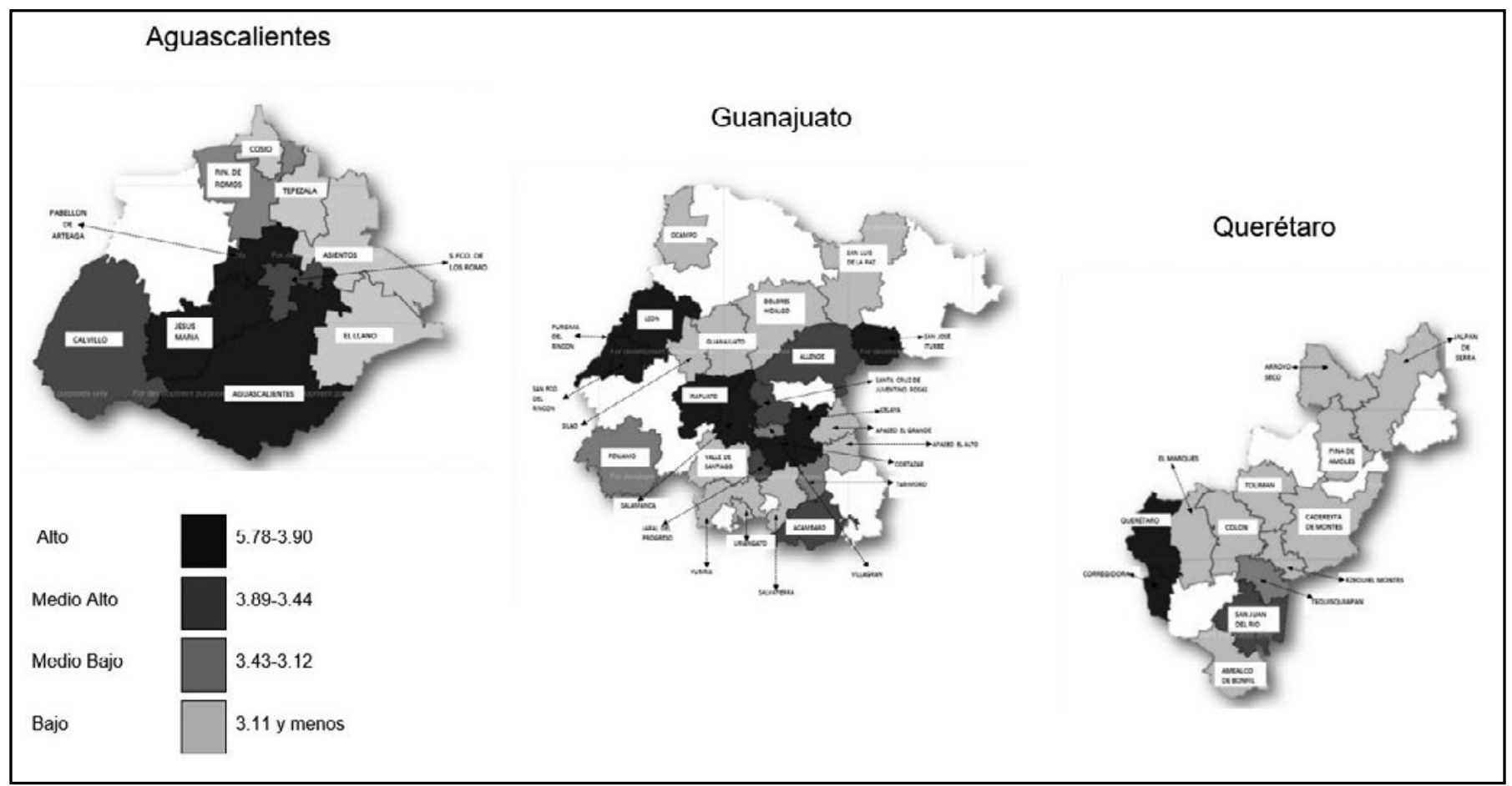

Figura 4. La brecha digital por municipios de los estados del Bajío (valor del IDT Mex, 2015). Elaboración propia con datos del INEGI (2016).

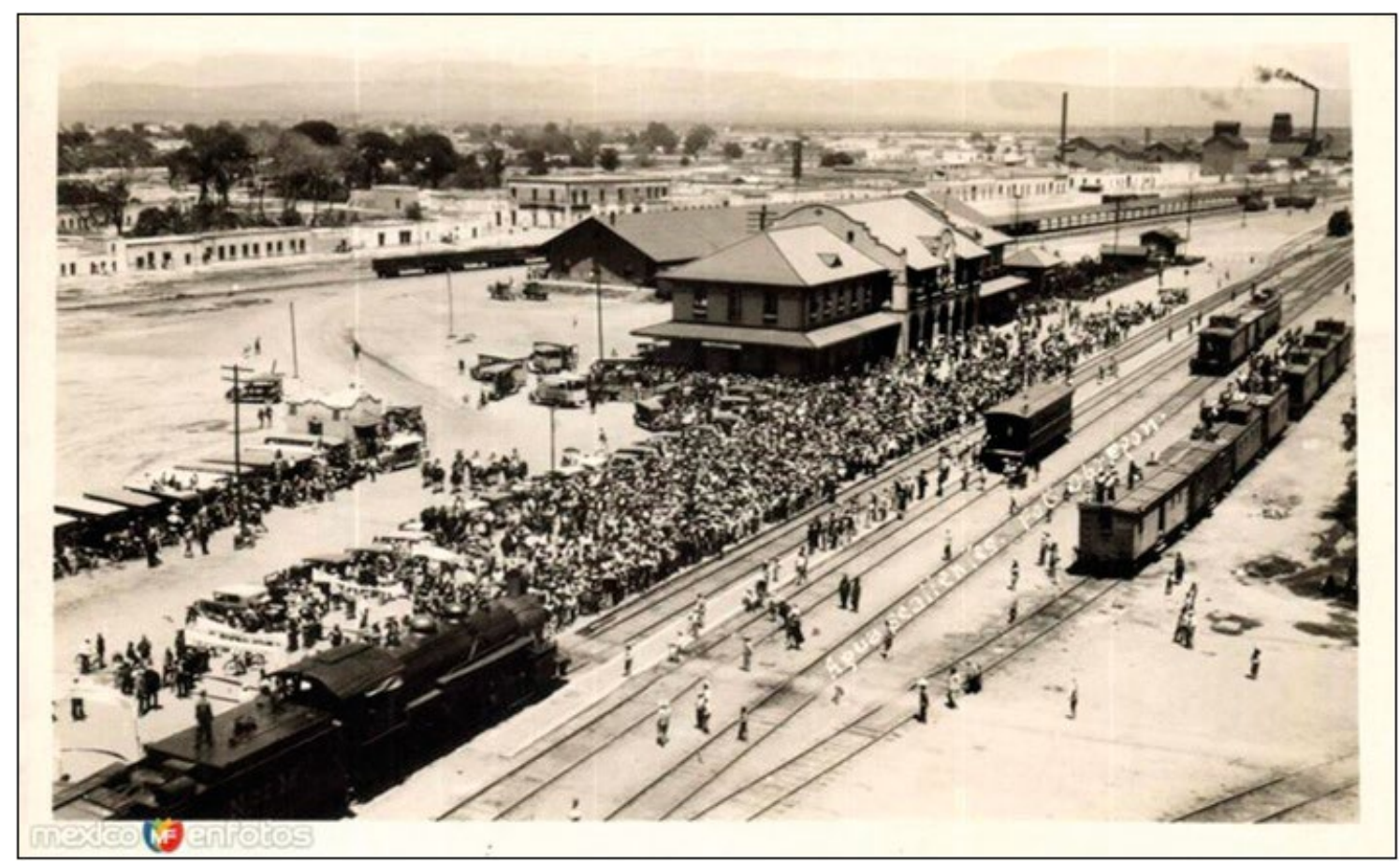

Figura 5. Estación del ferrocarril de Aguascalientes.

Fotografía tomada de villasanacarlos (17 de enero de 2012). 
CONCLUSIONES

Este trabajo es esencialmente un ejercicio de construcción de indicadores y análisis de los mismos que cubre un objetivo de medición de la brecha digital en los estados y municipios del Bajío, en el marco de las transformaciones estructurales de su economía. Pretende brindar una base para posteriores ejercicios de investigación que expliquen las condiciones meso y microeconómicas de la digitalización y el cambio estructural en los estados considerados. Es la vía de análisis de la modernización económica regional y del conocimiento de la nueva geografía económica mexicana, construida durante la fase del modelo neoliberal de los últimos 30 años.

Los tres estados del Bajío son ciertamente ejemplos deléxito en la atracción de IED; sin embargo, el despliegue de las características de su economía regional muestra resultados que son inferiores en algunos casos a los valores medios nacionales. En este caso, hemos visto una digitalización que no sobresale del nivel medio nacional y que incluso es más baja en el caso de Querétaro. En materia de crecimiento manufacturero los tres estados muestran una estabilidad que los sitúa sin cambios en el comparativo nacional.

En cambio, es en los servicios avanzados en donde Guanajuato y Querétaro muestran dinamismo superior al nacional y Aguascalientes tiene un pobre desempeño. En los tres estados la estructura de su producto ha variado con un descenso de la manufactura (que sigue siendo dominante) y un ascenso de servicios avanzados. El nivel medio de desarrollo del cambio estructural que muestran los estados parece brindar coherencia con el propio nivel de la digitalización que les corresponde, tal como es esperado dada la relación positiva entre el IDT Mex y el peso de los servicios avanzados en las economías regionales. La excepcionalidad que representa Querétaro, que tiene un bajo nivel de digitalización a pesar de su alto crecimiento de servicios avanzados, puede deberse al patrón de densidad de su digitalización en una parte del estado, la que contiene a la zona metropolitana de la capital; fenómeno que a su vez denota la polarización de su desarrollo urbano y económico en una clara diferencia con los otros estados analizados. Estas observaciones tienen carácter deductivo y en todo caso el valor de la información construida bajo la metodología mostrada y la caracterización del proceso de cambio estructural en el Bajío constituyen las aportaciones centrales del artículo.

REFERENCIAS

- $\quad \mathrm{AMIPCl}(2016)$. Estudio sobre los hábitos de internet en México 2016 [Presentación de Power Point]. Recuperada de https:// blog.elogia.net/estudio-sobre-los-habitos-de-los-usuarios-deinternet-en-mexico-2016

- Bataillon, C. (1969). Las regiones geográficas en México. México: Siglo XXI.

- $\quad$ Bell, D. (1973). The coming of post-industrial society. New York: Basic Books.

- Brynjolfsson, E., \& McAffe, A. (2014). The second machine Age. Boston: WW Norton \& Company.

- CEPAl (2016). La nueva revolución digital. De la internet del consumo a la internet de la producción. Santiago de Chile: Autor. Recuperado de https://www.cepal.org/es/ publicaciones/38604-la-nueva-revolucion-digital-la-internetconsumo-la-internet-la-produccion

- Daville-Landero, S. (2012). La evolución de la industria de autopartes en Querétaro, 1993-2008. Economía, sociedad y territorio, 12(40), 689-727.

- Esping-Andersen, G. (1999). Fundamentos sociales de las economías postindustriales. Barcelona: Ariel.

- El Observatorio Económico México Cómo Vamos, A. C. (2020). En cifras, ¿̇cómo vamos? México: Autor. Recuperado de https://mexicocomovamos.mx/encifrascomovamos.pdf

- Garza, G. (Coordinador) (2006). La organización espacial del sector servicios en México. México, D. F.: El Colegio de México.

- Gutiérrez Castorena, D., \& Gutiérrez Castorena, P. (2006). Dinámica industrial de Aguascalientes (1995-2000). Investigación y Ciencia de la Universidad Autónoma de Aguascalientes, 14(34), 42-50.

- Instituto Nacional de Estadística y Geografía. (1999). Censos Económicos 1999. México: Autor. Recuperado de https:// www.inegi.org.mx/programas/ce/1999/

(2004). Censos Económicos 2004. Recuperado de https://www.inegi.org.mx/programas/ce/2004/

(2007). Sistema de clasificación industrial de América del Norte (SCIAN). Recuperado de https://www. inegi.org.mx/rnm/index.php/catalog/1/download/1411 
(2009). Censos Económicos 2009. Recuperado de https://www.inegi.org.mx/programas/ce/2009/ (2014). Censos Económicos 2014. Recuperado de https://www.inegi.org.mx/programas/ce/2014/

(2015). Panorama sociodemográfico de México 2015. Recuperado de http://internet.contenidos.inegi.org. $\mathrm{mx} /$ contenidos/Productos/prod_serv/contenidos/espanol/ bvinegi/productos/nueva_estruc/702825078065.pdf (2016). Encuesta Nacional sobre Disponibilidad y Uso de Tecnologías de la Información en los Hogares 2015. Recuperado de https://www.inegi.org.mx/programas/ dutih/2015/

- Unión Internacional de Telecomunicaciones. (2014). Informe sobre medición de la sociedad de la información 2014. Resumen ejecutivo. Ginebra, Suiza: Autor.

- Mattelart, A. (2009). Histoire de la société de l'information. Paris: La Découverte.

- Micheli-Thirión, J. (2019). Cambio estructural en los estados especializados en producción automotriz de México, 1998-2013. Región y Sociedad, 31, el110. doi: 10.22198/ rys $2019 / 31 / 1110$

- Micheli-Thirión, J., \& Valle-Zárate, J. E. (2018). La brecha digital y la importancia de las tecnologías de la información y la comunicación en las economías regionales de México. Realidad, datos y espacio. Revista Internacional de Estadística y Geografía, 9(2), 38-53. Recuperado de https://www.inegi. org. $\mathrm{mx} / \mathrm{rde} / 2018 / 11 / 07 /$ la-brecha-digital-la-importancialas-tecnologias-la-informacion-la-comunicacion-en-laseconomias-regionales-mexico/

- Organisation for Economic Co-operation and Development.
(2000). The Service Economy. En Business and Industry Policy Forum Series. Paris: OECD.

- Salmerón Castro, F. I. (1998). Intermediarios del progreso. Política y crecimiento económico en Aguascalientes (282 pp.). México: CIESAS.

- Secretaría de Gobernación-Secretaría de Desarrollo Agrario, Territorial y Urbano. (2018). Sistema Urbano Nacional 2018. México: Autor. Recuperado de https://www.gob.mx/cms/ uploads/attachment/file/400771/SUN_2018.pdf

- Toudert, D. (2013). La brecha digital en los contextos de marginación socioterritorial en localidades mexicanas: Exploración y discusión. Comunicación y sociedad, 19, 153180. Recuperado de http://www.comunicacionysociedad. cucsh.udg.mx/index.php/comsoc/article/view/207/242

- Unger-Rubín, K. F. (2011). Competitividad y especialización de la economía de Guanajuato: Un acercamiento municipal, 1993-2003. Economía, Sociedad y Territorio, 11 (36), 403-454. Recuperado de http://www.scielo.org.mx/pdf/est/v11n36/ v1 $\ln 36 a 6 . p d f$

- villasanacarlos. (17 de enero de 2012). Estación del ferrocarril de Aguascalientes [Fotografía en MéxicoEnFotos]. Recuperada de https://www.mexicoenfotos.com/antiguas/ aguascalientes/aguascalientes/estacion-del-ferrocarril-deaguascalientes-MX13229838200581 


\section{Anexo}

El IDT Mex es un índice que se compone de diez factores agrupados en tres dimensiones (Micheli-
Thirión, \& Valle-Zárate, 2018), como se muestra en el listado siguiente:

\begin{tabular}{|c|c|}
\hline Dimensión & Factor \\
\hline \multirow[t]{4}{*}{ Acceso a TIC } & \% de hogares con telefonía fija \\
\hline & $\begin{array}{l}\text { \% de hogares con acceso a teléfono } \\
\text { inteligente }\end{array}$ \\
\hline & $\begin{array}{l}\text { \% de hogares con computadora de } \\
\text { escritorio o portátil }\end{array}$ \\
\hline & \% de hogares con acceso a Internet \\
\hline \multirow[t]{3}{*}{ Utilización de TIC } & \% de población que utiliza Internet \\
\hline & \% de población con conexión alámbrica \\
\hline & $\begin{array}{l}\text { \% de población con conexión } \\
\text { inalámbrica }\end{array}$ \\
\hline \multirow[t]{3}{*}{ Capacidades de uso de TIC } & \% de adultos alfabetizados \\
\hline & $\begin{array}{l}\text { \% de población mayor a } 18 \text { años con } \\
\text { estudios de bachillerato }\end{array}$ \\
\hline & $\begin{array}{l}5 \text { de población mayor a } 23 \text { años con } \\
\text { estudios de nivel superior }\end{array}$ \\
\hline
\end{tabular}

Para operacionalizar estos factores y calcular el IDT Mex se utilizaron las siguientes ecuaciones:

-La ecuación 1 fue utilizada para los primeros cuatro (Acceso) y la ecuación 2 para los otros seis (Utilización y Capacidades).

$$
s f_{m}^{k}=\frac{\sum_{1}^{n} H_{n}^{k} * F E_{n}^{H} \mid k=1}{\sum_{1}^{n} H_{n} * F E_{n}^{H}} ; \forall H_{n} \in m
$$

$$
s f_{m}^{k}=\frac{\sum_{1}^{n} P_{n}^{k} * F E_{n}^{P} \mid k=1}{\sum_{1}^{n} P_{n} * F E_{n}^{P}} ; \forall P_{n} \in m
$$


en donde:

$s f_{m}^{k}: s u b$ factor de característica $k$ en el municipio $m$

$k$ : Variable dicotómica con 1 como respuesta afirmativa.

$H_{n}^{k}:$ Hogar de la muestra $n$ que presenta la característica $k$

$F E_{n}^{H}:$ Factor de expansión correspondiente al hogar encuestado $n$

$H_{n}:$ Hogar encuestado de la muestra $n$

$P_{n}^{k}:$ Habitante de la muestra n que presenta la característica $k$

$F E_{n}^{P}:$ Factor de expansión correspondiente al habitante encuestado $n$

$P_{n}:$ Habitante encuestado de la muestra $n$

Con los valores obtenidos de los subfactores se calcularon los factores para cada municipio $m$ mediante la expresión 3.

$$
F_{m}^{K}=\sum_{1}^{k}\left(\frac{s f_{m}^{k} * w_{k}}{1000}\right)
$$

en donde:

$F_{m}^{K}:$ Factor del conjunto de caracteristicas $k$ en el municipio $m$

K: Conjunto de características evaluadas: Acceso, Utilización y Capacidades

$w_{k}$ :Peso de cada cacterística $k$ en $F^{K}$

A partir de los factores, se obtuvo el IDTMex para cada municipio, de la siguiente manera:

$$
\operatorname{IDTMex}_{m}=\sum_{1}^{K} F_{m}^{K} * \frac{W_{K}}{10}
$$

en donde:

$$
W_{K}: \text { Peso del factor } K \text { en el IDTMex }
$$

Finalmente, se calcula el indicador para cada una de las entidades del país (e) usando la expresión 5 .

$$
\operatorname{IDTMex}_{\boldsymbol{e}}=\frac{1}{m} \sum_{1}^{m} \operatorname{IDTMex}_{m} \mid m \in e
$$

